\title{
Modelling Method of Organization Internal Standard-complex
}

\author{
http://dx.doi.org/10.3991/ijoe.v11i7.4768 \\ Z.Feng ${ }^{1,2}$, F.Deng ${ }^{1}$, X.Luo ${ }^{2}$, M.Peng ${ }^{2}$ \\ ${ }^{1}$ College of Automation Science and Engineering, South China University of Technology, Guangzhou, China. \\ 2 Guangzhou Institute of Standardization, Guangzhou, China
}

\begin{abstract}
Organization is the main executive of standard implementation. Organization standard-complex can ensure the realization of organization functions by normalizing the mission of its members. This paper analyzes the control effect of the organization standard-complex to the achievements of organization functions. On this basis, this paper establishes a mathematic model of the planning process of organization standard-complex, and thus puts forward the structured planning method and steps.
\end{abstract}

Key words- Standard-complex, Standardization, Organization, Mathematical modelling

\section{INTRODUCTION}

Complex standardization is a standard activity that establishes a standard-complex and implements it. Complex standardization can upgrade the standardization from individual-level to system-level. Making use of the complex standardization method to transform the current standard system is an efficiency way to improve the standardization of enterprise level and make it adapt to modern society. The standard-complex in complex standardization refers to the object of complex standardization and the correlative elements, according to their intrinsic links or functionality requires, and forms a complete set of coordinated and optimal standards. Although the planning of standard-complex is the core work of complex standardization, there is seldom article about how to establish the systemic analysis method of the standard-complex and how to solve the related problems of the complex standardization.

Based on the requirement of the systemative method on the standard-complex planning, this paper presents a clear modeling method and step, then establishes the mapping relationship between the target and standard-complex to guarantee the sensibility to the change of the target.

\section{PRINCIPLE AND FRAMEWORK OF STANDARD-COMPLEX}

Standardization is an activity that develop, publish and implement standards for the best orders of things in a certain range. "A certain range" shows the boundedness of the standardization object, which means that outside the range is the environment of the object. The material, energy and information, received by the standardization object from environment, constitute the input, while those material, energy and information, outputted by the standardization object to the environment, constitute the output.
As a kind of information, the main function of standard is to make the output of standardization object be more suitable for the target (which means better effect). Therefore, it is not necessary for those things without targets to become the standardization object. According to the effect and efficiency change of the standardization object after the input of standard information, it is able to measure the effect of standardization. The effect of standard to standardization object has been shown as Figure 1.

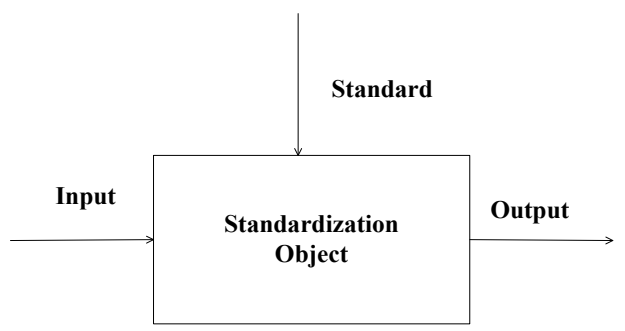

Figure 1. Diagram of the effect of standard to standardization object

In figure 1 , the standardization object is a connection between the input and output. Standard plays a promotion role to the standardization object but not the input. Inside the standardization, there are some corresponding modules to optimize the standard information input. This is the behavior to control the input and output based on the standard information, so this component is the standard controller. Existing a standardization controller is a necessary condition for standard information affecting the standardization object.

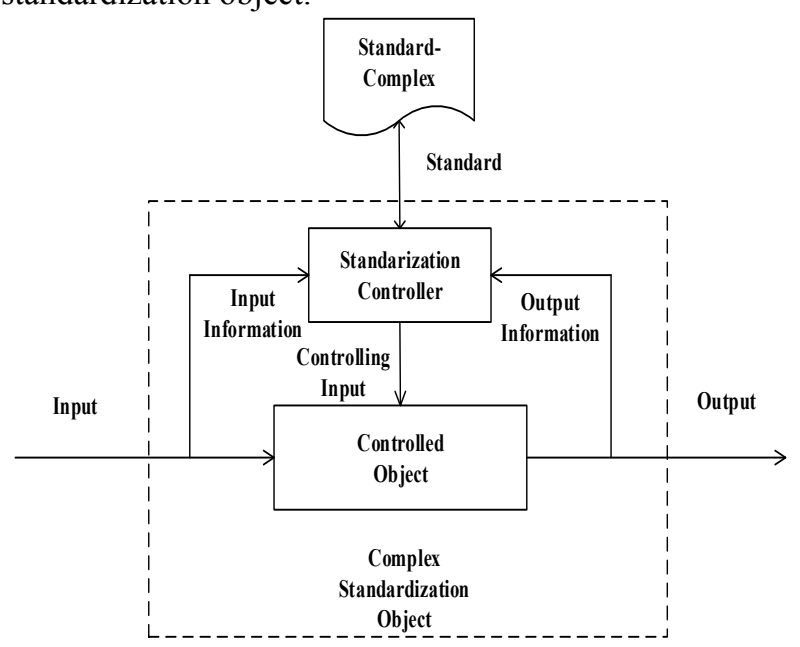

Figure 2. Concept diagram of standard-complex controller 
In complex standardization, the controller must control the standard-complex according to the object information, and change the standard-complex based on the changing of input, target and object. Therefore, the controller of complex standardization object has a two-way control effect to both itself and the standard-complex. The decomposition of interaction relationship is shown in Fig.2.

Fig. 3 is the working flow chart of standard controller. After the process of standardization, controller obtains the input information of standard object, and it checks the input information based on the input checking standard. For those input who have a normal result, they will go through the function achievement module to the controlled object and produce output. Then the controller will check the obtained output information based on the output checking standards. If the result of input or output checking is abnormal, the controller will go through the abnormal dispose controller based on the abnormal dispose standard.

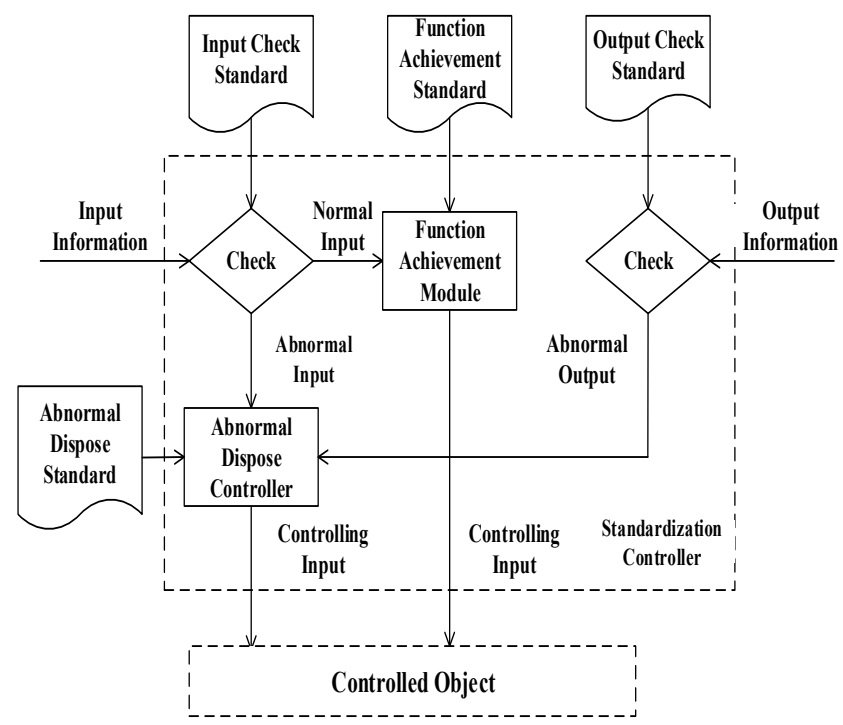

Figure 3. Work flow diagram of organization standard-complex

From the supporting relationship of standard to control process, we can obtain the top level frame of standardcomplex.

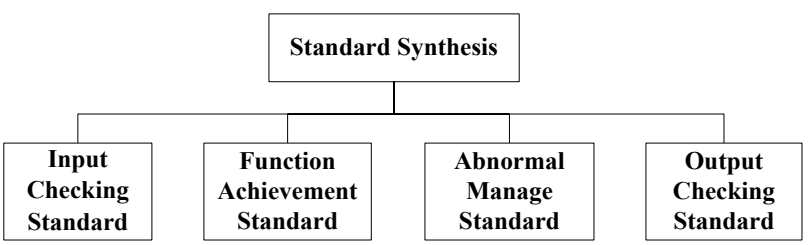

Figure 4. The top level frame of standard-complex

Fig.4 shows the top level frame of standard-complex. Based on Fig.4, we can have some further research combined with the standardization object as the control target to achieve the standard-complex planning diagram for the standardization object.

Next, we will research a diagram planning method for a standard-complex, which takes organization as the standardization object.

\section{BASIC PRINCIPLE OF CONSTRUCTING}

\section{A. According to the Target}

Standard-complex planning is a standardization activity with strong purpose. It needs to analyze the requirements to clear the control target of complex standardization object. Plan the standards under the goal-orientation is necessary.

\section{B. Control Function}

From previous analysis about standard-complex, theoretically the function of standard-complex is controlling. Especially when the complex standard object is an organization, standard is the operating rule of organization and the standard-complex is the blueprint to achieve the organization function.

\section{User-oriented}

User is the valuator of the standardization object operation. The user requirements analysis is the key mission in the process of standard-complex planning. Moreover, the participation and acceptance of users are the reflection of standard-complex.

\section{From Top Level to Bottom Level}

In the system analysis process, it needs to analyze the complex standardization object in a global perspective. Through the analysis from top level to bottom level, from roughness to delicate, from the outside to the inside, the standard planning module establishment should be step by step. Then reverse the process and form the planning model of standard-complex.

\section{Structured PlanNing Method of Standard- COMPLEX}

Organization refers to a collective or group that people get together to achieve a certain goal or collaborate with each other, such as the party organization, labor union organization, enterprise, military organization and so on. The standardization object of organization standardcomplex should be the organization in a narrow sense. Organization standard-complex can ensure the realization of organization functions and promote the achievement of organization goal by normalizing the mission of its members. An organization standard-complex includes a series of standards and documents. It can receive the twice the result with half the effort if we can have a systemative and perfect planning at the beginning of construction. There are three questions for the planning of standardcomplex:

What kind of standards do we need to establish?

What do these standards use for?

What situation do these standards suits?

\section{A. V mode Achieving Process of Standard-complex}

According to the standard-complex overall framework, the $\mathrm{V}$ mode achieving process of standard-complex is shown as Fig.5. 


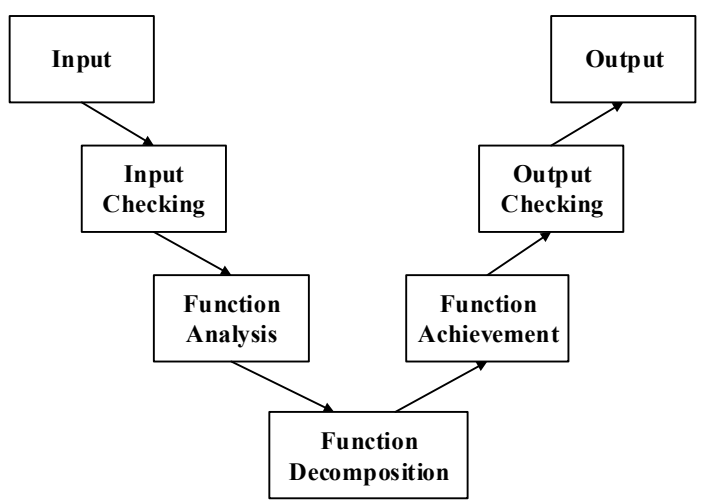

Figure 5. V mode achieving process of standard-complex

The input checking and output checking should turn to abnormal situation manage when there is an abnormal situation.

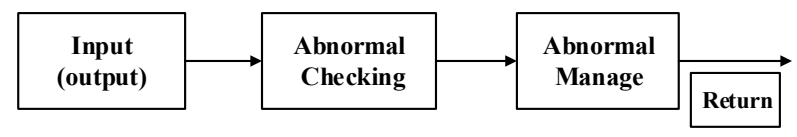

Figure 6. Abnormal situation manage process

\section{B. Structured matrix of standard-complex planning}

For convenience, the mathematical modelling process of organization standard-complex planning is shown as below:

Assuming $x_{k i}, i=1,2, \mathrm{~L}, m ; j=1,2, \mathrm{~L}, l$ are the input data, $y_{j}, j=1,2, \mathrm{~L}, l$ are the output data, we can construct a relevant matrix

$$
A=\left(\begin{array}{llll}
a_{11} & a_{12} & \mathrm{~L} & a_{1 l} \\
a_{21} & a_{22} & \mathrm{~L} & a_{2 l} \\
\mathrm{~L} & \mathrm{~L} & \mathrm{~L} & \mathrm{~L} \\
a_{m 1} & a_{m 2} & \mathrm{~L} & a_{m l}
\end{array}\right)
$$

Among this, the matrix factor

$$
a_{i j}=\left\{\begin{array}{l}
1, x_{i} \rightarrow y_{i} \\
0, \neg x_{i} \rightarrow y_{i}
\end{array} i=1,2, \mathrm{~L}, m ; j=1,2, \mathrm{~L}, l\right.
$$

$\mathrm{A}$ is the relevant matrix of input and output.

We can construct the augmented matrix $\bar{A}$ :

$$
\bar{A}=\left(\begin{array}{cc}
0 & Y^{\prime} \\
X & A
\end{array}\right)
$$

Among this, $\quad X=\left(\begin{array}{llll}x_{1} & x_{2} & \mathrm{~L} & x_{m}\end{array}\right)^{\prime} \quad$,

$Y=\left(\begin{array}{llll}y_{1} & y_{2} & \mathrm{~L} & y_{l}\end{array}\right)^{\prime}$ are column vectors.

$\bar{A}$ is the structure transformation matrix.

For the structure transformation matrix $\bar{A}=\left(\begin{array}{cc}0 & Y^{\prime} \\ X & A\end{array}\right)$

a. Do elementary row transformation except the first row: exchange the position of two rows.

b. Do elementary column transformation except the first column: exchange the position of two column.
Let the factor which is valued equals 1 to be close to the diagonal and we can have the matrix with the following structure:

$$
\bar{A}^{*}=\left(\begin{array}{cc}
0 & Y^{* \prime} \\
X^{*} & A^{*}
\end{array}\right)
$$

Among this, $X^{*}=\left(\begin{array}{lll}x_{i 1} & \mathrm{~L} & x_{i m}\end{array}\right)^{\prime}$ is an arrangement of $\mathrm{X}$ factors, $Y^{*}=\left(\begin{array}{lll}y_{i 1} & \mathrm{~L} & y_{i l}\end{array}\right)^{\prime}$ is an arrangement of $\mathrm{Y}$ factors.

$$
A^{*}=\left(\begin{array}{cccc}
A_{1} & & & \\
& A_{2} & & \\
& & \mathrm{~L} & \\
& & & A_{s}
\end{array}\right)_{m \times l}+B_{m \times l}
$$

In this matrix, $A_{1}, \mathrm{~L}, A_{s}$ are block matrixes. $B$ is the matrix when all the factors of $A_{1}, A_{2}, \mathrm{~L}, A_{s}$ are substituted by 0 in $A^{*}$.

We can say $A^{*}$ is the function treatment structured matrix. Obviously $A^{*}$ is the similar matrix of $A, B$ is the sparse matrix.

Take $A_{k}, \quad k=1, \mathrm{~L}, s$ as the basic functional module to decompose the organization function. This is the basis of organization standard-complex planning.

Assuming $\quad f=\left(\begin{array}{l}f_{1} \\ \mathrm{M} \\ f_{l}\end{array}\right) \quad, \quad$ which satisfies $Y^{*}=f\left(A^{*} X^{*}\right)=f\left(\begin{array}{c}A_{1} X_{1} \\ \mathrm{M} \\ A_{s} X_{s}\end{array}\right)+f\left(B X^{*}\right)$

Then $f=\left(f_{1}, \mathrm{~L}, f_{l}\right)$ is the function manage function, $y_{j}=f_{j}\left(\sum a_{i j} x_{i}\right) \quad j=1, \mathrm{~L}, s, X_{k}$ is a series of corresponding input of $A_{k}$.

\section{Steps of structured planning method for organization standard-complex}

From the previous analysis, the structure planning steps of an organization standard-complex is as following:

1) Construct the $\mathrm{V}$ mode of achieving process according to the target of standardization object;

2) According to the $V$ mode and organization target, recognize the output vector $Y=\left(y_{1}, \mathrm{~L}, y_{l}\right)^{\prime}$, and input vector $X=\left(x_{1}, \mathrm{~L}, x_{m}\right)$

3) According to the relationship of input and output, recognize the relevant matrix $A=\left(a_{i j}\right)_{m \times l}$.

4) In the standardization object implementation process, identify the manage function $f=\left(f_{1}, \mathrm{~L}, f_{l}\right)$.

And make

$$
Y=f(A X)
$$

Which means 


$$
y_{j}=f_{j}\left(\sum_{i=1}^{m} a_{i j} x_{i}\right)
$$

5) Do the elementary transformation to $\bar{A}=\left(\begin{array}{ll}0 & Y^{\prime} \\ X & A\end{array}\right)$, let the factors in $A$ which equals 1 to be close to the diagonal.

Then we can obtain the matrix $\bar{A}^{*}=\left(\begin{array}{cc}0 & Y^{* \prime} \\ X^{*} & A^{*}\end{array}\right)$;

6) Take $A_{k}, \quad k=1, \mathrm{~L}, S$ as the basic functional module to decompose the organization function. Design the standard-complex according to the module after decomposition.

\section{CONCLUSION}

From the micro view of standardization activity implementation, this paper researched the work principle of standard-complex which takes the organization as the complex standardization object, and analyzed the control effect of organization standard-complex to organization function achievement. Based on this, this paper constructed a mathematical model of organization standard-complex, and put forward the structured planning method and steps.

The content of this paper are the supplement and improvement of the systemative method about Complex standardization object target analysis and standardcomplex planning in Chinese national standard $<$ Guideline for the word of Complex standardization> (GB 12366). It extends the application area of Complex standardization method from products to organizations. It enhances the operation ability of Complex standardization method implementation, which can improve the effect of Complex standardization method in promoting standard efficiency.

\section{REFERENCES}

[1] C.Li, "The characteristics of complex standardization", in Guide Paper of China standard, pp. July, 2011

[2] X.Dong, Z.Zhao, Z.Peng and etc., "Guideline for the word of complex stnadardization", China National Institute of Standardization, 2009.

[3] F.Han, W.Tang, Z,Chen and etc., "Criteria for performance excellence", China National Institute of Standardization, 2012.

[4] H.Cheng, Y.Liu, "The convergence of interests standard theoretical framework and system innovation," in Macro Quality Research, October 2013.

[5] Z.Peng, D.Bai, Y.Xu and etc., "Guide for standardization - Part 1: Standardization and related activities - General vocabulary," China National Institute of Standardization, 2009.

[6] G.Yue, L.Xing, Z.Zhao and etc., "Principles and requirements for preparing diagrams of standard system," China National Institute of Standardization, 2009.

[7] C.Li, "The connection of system science and standardization Dicussion on the scientific value of Complex standardization" in Standard Science, Mar,2009

\section{AUTHORS}

Zhihui.Feng, is currently a doctor student in South China University of Technology. He is also the vice president of Guangzhou Institute of standardization (email: qbsfzh@gmail.com).

Feiqi.Deng, is the corresponding author of this paper. $\mathrm{He}$ is now the president of System Engineering Institute of South China University of Technology (e-mail: aufqdeng@scut.edu.cn).

Xuan.Luo, is a researcher of Guangzhou Institute of standardization (e-mail: luoxuan1988@gmail.com).

Mianli.Peng, is a researcher of Guangzhou Institute of standardization (e-mail: gd_ecs@163.com).

Submitted 25 May 2015. Published as resubmitted by the author 25 june 2015. 